\title{
Orientações para utilização de acelerômetros no Brasil
}

\author{
Instructions for accelerometer use in Brazil
}

\begin{abstract}
Jeffer Eidi Sasaki', Andrée Philippe Pimentel Coutinho², Carla Elane Silva dos Santos², Cecília Bertuol², Giseli Minatto², Juliane Berria², Lúcia Midori Damaceno Tonosaki², Luiz Rodrigo Augustemak de Lima², Moane Marchesan², Pablo Magno da Silveira², Rodrigo de Rosso Krug², Tânia R. Bertoldo Benedetti²
\end{abstract}

\section{Resumo}

O objetivo do presente estudo foi apresentar um conjunto de orientações para a utilização de acelerômetros no Brasil. O método consistiu na revisão e síntese de artigos que utilizaram acelerômetros como instrumento de medida objetiva da atividade física. Com base nesses estudos, buscou-se apresentar orientações para as fases pré-coleta (seleção do acelerômetro; testagem da calibração dos acelerômetros, atualização de firmware; estudo piloto; e definição de protocolo), coleta (distribuição dos acelerômetros; contato com participantes; e devolução dos acelerômetros) e pós-coleta (processamento; transformação e interpretação dos dados; e comparabilidade dos dados) do uso de acelerômetros. São descritos procedimentos para facilitar as tomadas de decisões relacionadas ao uso desses dispositivos, bem como para obtenção de dados de acelerometria válidos e reprodutíveis. As orientações são uma iniciativa de pesquisadores da área a fim de proporcionar um avanço metodológico nas medidas objetivas da atividade física. A adoção das orientações pode facilitar a padronização dos procedimentos empregados e a comparabilidade de resultados entre estudos com acelerômetros no Brasil.

\section{Palavras-chave}

Monitoramento; atividade motora; métodos; avaliação.

\begin{abstract}
The aim of this study was to develop instructions for using accelerometers to measure physical activity in Brazil. This manuscript is a review and synthesis of scientific papers that have used accelerometers for objective physical activity assessment in different age groups as well as studies providing directions for using activity monitors in large-scale studies. In this study, we present instructions for the pre-data collection (selection of monitor; unit calibration, firmware update, pilot study, protocol definition), data collection (monitor distribution, contacting participants, monitor return) and post-data collection (data processing, transformation and interpretation) phases. We describe procedures for facilitating decision making related to using accelerometers, as well as for obtaining valid and reliable accelerometer physical activity data. This set of instructions is an initiative of a group of physical activity researchers with the purpose of contributing to methodologically advance the field of objective physical activity measurement in Brazil. The current set of instructions intend to facilitate the standardization of procedures for collecting physical activity data with accelerometers in Brazil and, thus, for ascertaining future comparability of data collected in different studies.
\end{abstract}

\section{Keywords}

Monitoring; Motor activity; Methods; evaluation.

\section{Introdução}

Os benefícios da prática habitual de atividade física (AF) para a saúde já estão evidenciados na literatura. Pessoas fisicamente ativas têm menor propensão de apresentar doenças crônicas não transmissíveis ${ }^{1}$. O estabelecimento dessas associações tem acontecido tanto pela estimativa da AF por meio de autorrelato como também por meio de sensores de movimento ${ }^{2,3}$,

1 Universidade do Triângulo Mineiro, Departamento de Ciências do Esporte, Programa de Pós-Graduação em Educação Física. Uberaba, Minas Gerais, Brasil.

2 Universidade Federal de Santa Catarina, Centro de Desporto, Programa de Pósgraduação em Educação Física. Florianópolis, Santa Catarina, Brasil. os quais têm ganhado atenção na comunidade científica devido ao maior nível de acurácia e precisão.

Entre os sensores de movimento capazes de mensurar a AF estão os acelerômetros, dispositivos eletrônicos que medem a aceleração do movimento corporal em um, dois ou três planos (anteroposterior, médiolateral e vertical) ${ }^{4}$. Esses equipamentos permitem quantificar objetivamente a frequência, duração e intensidade da AF em função das características dos sinais de aceleração, como o padrão de oscilação, o intervalo de tempo e a magnitude dos mesmos ${ }^{4}$. Em geral, os dados coletados com acelerômetros apresentam maior 
nível de validade e reprodutibilidade do que instrumentos de autorrelato, fatores importantes para estabelecer relações dose-resposta com variáveis de saúde $e^{5,6}$.

Embora os acelerômetros sejam crescentemente utilizados em estudos de larga escala ${ }^{7-9}$, revisões sistemáticas indicam a necessidade de padronização nos métodos de mensuração da AF empregados em estudos no Brasil, sejam de autorrelato ou objeti$\operatorname{vos}^{10,11}$. A padronização dos métodos de mensuração da AF possibilita maior comparabilidade de resultados entre diferentes estudos, o que é importante no estabelecimento e consolidação das associações entre a AF e desfechos de saúde ${ }^{12}$.

Neste sentido, diversos fatores relacionados ao uso de acelerômetros merecem atenção para que permitam comparabilidade entre diferentes estudos. O presente artigo aborda questões relacionadas as fases pré-coleta (seleção do acelerômetro; testagem da calibração dos acelerômetros, atualização de firmware; estudo piloto; e definição de protocolo), coleta (distribuição dos acelerômetros; contato com participantes; e devolução dos acelerômetros) e pós-coleta (processamento; e transformação e interpretação dos dados) da utilização de acelerômetros. Alguns procedimentos podem facilitar as tomadas de decisões relacionadas a as referidas etapas de utilização dos acelerômetros, bem como a obtenção de dados válidos e reprodutíveis, permitindo maior comparabilidade entre estudos. Assim, o objetivo deste estudo foi apresentar um conjunto de orientações para utilização de acelerômetros no Brasil.

\section{Fase pré-coleta}

A fase pré-coleta consiste no momento de definição dos aspectos físicos e logísticos da utilização do acelerômetro. Envolve ainda o planejamento do protocolo de estudo e sua testagem. O intuito dessa seção não é abordar todos os aspectos da fase pré-coleta, mas sim alguns pontos principais que podem auxiliar os pesquisadores da área. Deste modo, os pontos abordados são a seleção do acelerômetro; testagem da calibragem dos acelerômetros; atualização de firmware; definição de protocolo (taxa de amostragem, posicionamento do acelerômetro e tempo de uso) e realização de estudo piloto.

- Seleção do acelerômetro: entre alguns dos modelos utilizados em pesquisa, é possível citar o Actical, RT6, ActiGraph GT3X+, GENEActiv, BodyMedia Armband e o ActivPAL. Uma breve descrição a respeito das especificações técnicas de alguns desses sensores de movimento pode ser encontrada no estudo de Strath et al. ${ }^{13}$ A seleção do acelerômetro baseia-se em geral nas necessidades de investigação ${ }^{14}$. Alguns fatores a serem considerados são a necessidade de coletar dados triaxiais, a magnitude de detecção da aceleração (por exemplo, $\pm 3 g$ vs $\pm 6 g$ ), bateria e memória, adequabilidade para medir o comportamento sedentário, viabilidade e comparabilidade com estudos anteriores.

Atualmente, a maioria dos acelerômetros comercializados são triaxiais, possuindo a capacidade de medir a aceleração nos três planos ortogonais: vertical, anteroposterior e médio-lateral. Porém, muitos pesquisadores continuam a utilizar acelerômetros uniaxiais em suas pesquisas ${ }^{13}$. Além do número de eixos de detecção, podem existir diferenças na filtragem proprietária entre acelerômetros de diferentes marcas, resultando em diferenças na frequência e magnitude de aceleração necessária para o registro de um count ${ }^{15}$. A sensibilidade na detecção da aceleração pode também ser influenciada pelo tipo de transdutor empregado, podendo ser piezoelétrico, piezorresistivo ou de capacitância diferencial ${ }^{4}$. Devido a variedade de características dos acelerômetros, não é pos- 
sível sugerir a utilização de uma determinada marca e modelo. É necessário que cada pesquisador avalie as características dos acelerômetros conforme o objetivo do estudo a ser conduzido.

Contudo, se a comparabilidade de dados é o principal objetivo do estudo, as principais questões a serem consideradas são: 1) Com qual estudo(s) deseja-se comparar os resultados? 2) Qual acelerômetro foi utilizado nesse(s) estudo(s)? 3) O acelerômetro ainda é comercializado? 4) Existe algum equivalente mais moderno? 5) O método aplicado para a derivação de estimativas de AF foi baseado em counts (por exemplo, pontos de corte) ou dados brutos (múltiplos de força g)? 6) Existem estudos demonstrando equivalência do acelerômetro utilizado em estudo prévio e o modelo que se pretende utilizar?

- Calibração dos acelerômetros: é fundamental que os pesquisadores verifiquem a calibragem dos acelerômetros antes de utilizá-los em qualquer estudo ${ }^{16}$. Esse procedimento é realizado para verificar o nível de confiabilidade no registro dos dados de aceleração ${ }^{16}$. Duas formas comuns de realizar o teste de calibragem são: 1) utilizar velocidades padrões na esteira (por exemplo, 4,8 $\mathrm{km}^{-1} \mathrm{~h}^{-1}, 6,4$ $\mathrm{km} \cdot \mathrm{h}^{-1}$ ) ou 2) utilizar um oscilador mecânico (shaker). Quando a primeira opção é selecionada, podem-se utilizar dois acelerômetros posicionados lado a lado em cada quadril. Para otimizar o processo de testagem, ainda é possível sobrepor unidades. O ideal é não sobrepor mais do que dois acelerômetros, pois esse procedimento pode resultar em artefato/ruído nos sinais. As diferentes unidades devem demonstrar valores semelhantes entre $\mathrm{si}^{17}$. É recomendável que os pesquisadores estabeleçam um nível aceitável de diferença entre as unidades, preferencialmente $<5 \%{ }^{17}$. Esse nível depende do estudo no qual os aparelhos serão utilizados. Por exemplo, se um determinado lote de acelerômetros sistematicamente registra dados $5 \%$ superiores a outro lote, torna-se evidente que os dois lotes não devem ser utilizados em uma intervenção de mudança na AF, pois haverá atenuação ou exacerbação dos reais efeitos da intervenção, a depender de qual lote for utilizado primeiro.

- Atualização de firmware: o firmware é um conjunto de instruções operacionais que é diretamente programado no hardware de equipamentos eletrônicos, nesse caso o acelerômetro ${ }^{18}$. Essas instruções operacionais possuem os comandos básicos para a detecção, registro e armazenamento de dados, bem como o funcionamento geral do equipamento. O firmware é atualizado sempre que o fabricante encontra problemas relacionados aos fatores citados. Contudo, o firmware pode impactar as estimativas dos acelerômetros e comprometer a comparabilidade entre diferentes estudos ${ }^{19,20}$.

Alguns cuidados podem evitar e/ou auxiliar na minimização de eventuais problemas gerados por atualizações de firmware, como:

- Atualizar os acelerômetros com o firmware mais recente antes de iniciar o estudo ${ }^{20}$;

- manter o mesmo firmware durante a coleta, evitando atualizar as versões enquanto a coleta estiver ocorrendo ${ }^{20}$;

- realizar inicialização e descarregamento dos acelerômetros na mesma versão de software;

- manter o registro $(\log )$ da atualização de firmware e/ou software e salvar o comunicado fornecido pelo fabricante.

- Posicionamento do acelerômetro: para mensuração da AF, os acelerômetros devem ser bem fixados no corpo do participante por meio de uma cinta (geralmente elástica) ou pulseira no local escolhido (por exemplo, quadril, punho). A 
correta fixação minimiza as vibrações e deslocamentos que acarretam ruído/ artefato na medida do movimento ${ }^{21}$. Os principais locais para utilização do acelerômetro são: quadril, punhos, região anterior e posterior da caixa torácica, coxas e tornozelo ${ }^{21,22}$. A maioria das pesquisas tem posicionado o acelerômetro no quadril dos participantes, devido à proximidade do centro de massa do corpo humano ${ }^{21-23}$. Assim, as acelerações medidas por um único sensor neste local podem representar melhor o gasto energético proveniente do movimento humano. O posicionamento no quadril resulta em baixo constrangimento aos avaliados e pouco desconforto no movimento corporal. Apesar de diversas atividades serem adequadamente medidas pelo acelerômetro no quadril, existem atividades predominantemente realizadas com os membros superiores e que frequentemente não são captadas por esse posicionamento ${ }^{21,24}$. O posicionamento do acelerômetro no punho tem sido adotado para aumentar a taxa de uso $^{6}$. Estudos internacionais em amostras populacionais representativas (por exemplo, National Health and Nutrition Examination Survey - NHANES) têm adotado a utilização do acelerômetro no punho e demonstrado um aumento na taxa de uso ${ }^{6}$. Porém, o número de métodos para processar dados coletados no punho ainda é escasso ${ }^{25}$. Por isso, a utilização do acelerômetro no punho ou quadril deve se basear na disponibilidade de métodos de processamento e na intenção de comparar dados com estudos passados ou futuros, e na possibilidade de aplicar métodos de aprendizado de máquina. Atualmente, o posicionamento do acelerômetro é uma decisão "no escuro", não havendo consenso sobre o mesmo.

- Taxa de amostragem: a tecnologia atual permite que os dados sejam coletados em sua forma bruta (múltiplos de força g) e em diferentes taxas de amostragem (por exemplo, 30, 40, $100 \mathrm{~Hz})^{21}$. Os acelerômetros da marca ActiGraph, por exemplo, têm uma capacidade atual de registrar dados a $100 \mathrm{~Hz}^{13}$. É recomendável que os pesquisadores coletem os dados em maiores frequências e reintegrem para frequências menores no período pós-coleta, haja vista que não é possível reintegrar dados de frequências menores para maiores ${ }^{21}$. Dessa forma, ao se coletar os dados em frequências maiores preservam-se as possibilidades de aplicação de novos métodos de processamento e a comparabilidade com estudos futuros.

- Número de dias e horas de coleta: para a coleta de dados, é necessário estipular os parâmetros relacionados ao tempo de uso do acelerômetro, como por exemplo, dias e horas/dia de uso. Esse tempo deve refletir a rotina habitual do avaliado. Tem sido usual a coleta de dados por pelo menos quatro dias (três dias de semana e um de fim de semana) para crianças e adolescentes, e três dias (dois dias de semana e um de fim de semana) para adultos ${ }^{22}$. Esses números consistem em valores mínimos para atingimento de um bom nível de confiabilidade. O cenário ideal é a coleta de dados por sete dias da semana ${ }^{22}$, o que permite verificar o atingimento das recomendações semanais de AF moderada-vigorosa sem utilizar métodos de imputação de dados. No entanto, a decisão de coletar dados por uma semana inteira pode ser difícil em estudos com alto número de participantes, sendo necessário considerar a viabilidade e o cronograma de coleta.

- Criação de diário de registro: a função do diário de registro é compilar todas as informações relacionadas às adversidades e situações vivenciadas pelos participantes da pesquisa. O diário de registro é importante na anotação de situações de retirada e colocação do aparelho, bem como as razões que resultaram em tais 
ações ${ }^{14,21}$. Essas instruções devem ser transmitidas aos participantes durante a visita de entrega dos acelerômetros. O diário de registro é um instrumento que auxilia na verificação do tipo de atividade realizada durante a não-utilização do acelerômetro e o objetivo da mesma (por exemplo, atividade aquática, esporte, evento social etc $)^{14}$. Desta forma, o diário de registro auxilia na interpretação dos dados do acelerômetro, tornando possível o seu ajuste quando necessário.

- Estudo piloto: o estudo piloto é uma prática comum em pesquisa. No contexto da acelerometria não deve ser diferente. O estudo piloto é fundamental para observação de situações que influenciam diretamente a obtenção dos dados, como o posicionamento do equipamento, as instruções para os participantes e a forma de manusear o aparelho. O estudo piloto permite antecipar as possíveis reações dos participantes acerca da utilização dos acelerômetros. Permite também examinar questões logísticas e operacionais da sua utilização, possibilitando ajustes no protocolo antes de iniciar o estudo. Nesta etapa, deve-se testar o protocolo de coleta de dados de forma semelhante ao que será implementado no estudo. Isso permite determinar se o protocolo, bem como os fatores físicos e eletrônicos dos acelerômetros, como a bateria e memória, estão adequados ao período de coleta.

\section{Fase de coleta dos dados}

A fase de coleta de dados é aqui definida como o período compreendido desde o momento da entrega dos acelerômetros até o seu recolhimento. Esse período constitui-se na oportunidade que os pesquisadores possuem de reforçar as instruções de uso (distribuição dos acelerômetros), questionar sobre a utilização efetiva do acelerômetro (contato telefônico com os participantes) e obter informações relevantes acerca de imprevistos ocorridos no período de uso (recolhimento dos acelerômetros).

- Distribuição dos acelerômetros: previamente à data marcada para a entrega dos acelerômetros, é importante verificar o material e suas condições de uso. Os acelerômetros devem estar previamente inicializados e com nível suficiente de bateria para o período de coleta. Vale ressaltar que diferentes modelos de acelerômetros possuem diferentes capacidades de bateria e que as configurações de amostragem (por exemplo, taxa de amostragem, ativação da função "idle mode") impactam no período de duração da mesma. O pesquisador precisa verificar se o acelerômetro escolhido e as configurações utilizadas se adequam ao período de coleta. Outro ponto fundamental é providenciar tamanhos de cintas elásticas distintos, pois uma cinta previamente separada pode não se ajustar às medidas do avaliado.

A estratégia para entregar os acelerômetros aos participantes depende dos custos, do desenho do estudo e do tamanho da amostra ${ }^{26}$. Os acelerômetros podem ser entregues por postagem ou face a face ${ }^{26}$. O envio dos acelerômetros por correio tem sido relativamente comum em estudos realizados em países desenvolvidos ${ }^{7,26,27}$, mas essa estratégia não tem sido adotada no Brasil. Portanto, a entrega face a face é uma estratégia mais aplicável à nossa realidade e possibilita a explicação dos procedimentos de uso e demonstração da correta colocação do acelerômetro, permitindo também responder as dúvidas dos avaliados $^{26}$. Durante a entrega do acelerômetro, o pesquisador deve enfatizar o período de utilização (por exemplo, 7 dias), tempo de uso diário (por exemplo, 8-10 h) e necessidade de retirar o dispositivo durante atividades aquáticas, no 
banho e à noite ao dormir (se tal período não é de interesse). As instruções para crianças e idosos devem ser em linguagem simples, assegurando a compreensão dos procedimentos a serem seguidos.

É importante entregar um material informativo impresso contendo as orientações aos participantes ${ }^{26}$. Neste contexto, fotos e/ou imagems ilustrando o posicionamento do aparelho no corpo podem facilitar a compreensão. Ainda assim, recomenda-se fornecer informações de contato pessoal (telefones e e-mails) aos participantes para que possam esclarecer dúvidas durante o período de utilização do aparelho ${ }^{26}$.

Para a organização e o gerenciamento da coleta de dados, convém criar um formulário para armazenar informações referentes aos acelerômetros e aos participantes, como o código de identificação do participante, a data de entrega, o número serial do acelerômetro e a data de início do registro das atividades.

Recomenda-se que o diário de registro seja entregue aos participantes juntamente com o aparelho. Os participantes devem registrar nesse diário os horários em que despertam, que se deitam, e quando colocam e retiram o apare$l_{\text {ho }}{ }^{21}$. Devem ser registrados também os períodos em que o aparelho não foi utilizado e as razões para tal (por exemplo, atividade aquática). Embora a utilização do diário de registro seja importante, a complexidade analítica dificulta a sua utilização em estudos epidemiológicos ${ }^{14}$.

- Contato com participantes: durante a coleta de dados, recomenda-se a realização de contato(s) com os participantes, podendo ser realizado(s) via telefone, e-mail ou $\mathrm{SMS}^{26}$. O ideal é que o contato seja realizado após um ou dois dias da entrega dos acelerômetros. O pesquisador deve perguntar sobre a utilização do dispositivo, o preenchimento do diário de registro, e reforçar as informações com relação ao número de dias e tempo de uso necessário para validar um dia habitual. Caso o participante relate não estar utilizando o acelerômetro corretamente, o pesquisador pode aproveitar esse contato para estender a data de entrega, possibilitando que o aparelho fique com o avaliado por mais dias, a fim de atingir o tempo de utilização estabelecido previamente.

- Devolução dos acelerômetros: a devolução do acelerômetro pode ser realizada em visita do participante ao laboratório, ou, do pesquisador ao domicílio do participante ${ }^{26}$. A forma de recuperação do acelerômetro deve ser previamente estabelecida, sempre considerando a conveniência e comodidade do participante. Durante a devolução, o pesquisador deve identificar se o avaliado encontrou dificuldades para a utilização do acelerômetro e conferir o preenchimento do diário de registro, solicitando ao avaliado que recorde informações faltantes para completar o diário. Logo após a devolução dos acelerômetros, é importante que o pesquisador faça o download dos dados, tendo em vista que o aparelho continua registrando informações, o que gera acúmulo desnecessário de dados, e pode sobrecarregar o armazenamento das informações ${ }^{21}$.

\section{Fase pós-coleta}

A fase pós-coleta envolve a limpeza, redução, manuseio e análise dos dados. Todas essas ações são peculiares aos objetivos de pesquisa e, portanto, diferem entre estudos. O intuito dessa seção é apresentar alguns procedimentos que merecem atenção por parte dos pesquisadores.

- Conduta imediata de processamento de dados: a etapa de processamento de dados tem três objetivos: 1) identificação bruta dos dados que podem não represen- 
tar com precisão o comportamento da AF; 2) decisão de como lidar com esses dados; e 3) preparação dos dados para análise estatística e interpretação. Os arquivos inadequados devem ser descritos em uma planilha com as razões para exclusão da análise. Isso pode auxiliar a manter uma organização para análises futuras ${ }^{28}$.

O pesquisador deve realizar a identificação dos dados discrepantes para o controle de qualidade, ou seja, valores muito altos ou muito baixos de acordo com limites conhecidos e esperados. Essa identificação é realizada por meio de técnicas e combinações quantitativas e qualitativas de avaliação. A verificação quantitativa comum é realizada na tela bruta dos dados, onde se identificam os pontos que excedem um limiar predeterminado (por exemplo não-existência de valores superiores a 11000 counts $/ \mathrm{min}^{29}$ ). Um método qualitativo eficaz inclui a inspeção visual de gráficos para identificar valores divergentes e períodos de não-uso ou de uso do aparelho demasiadamente longos. O controle de qualidade também inclui a identificação dos requisitos mínimos para validar o dia de uso (por exemplo, dias com o mínimo de 10 horas; 4 dias de uso, sendo um dia de final de semana).

- Transformação de dados: os dados brutos do acelerômetro são normalmente transformados em uma ou mais variáveis fisiológicas e/ou comportamentais. Essas transformações utilizam uma variedade de técnicas analíticas, exemplificadas pela regressão linear simples ${ }^{19,30}$, pela regressão múltipla ${ }^{31}$ e pelos algoritmos de aprendizado de máquina ${ }^{32-36}$. Esses algoritmos utilizam os dados brutos (força g) ou counts do acelerômetro para estimar o gasto energético, a intensidade ou o até mesmo o tipo de atividade (por exemplo, locomoção, esporte, atividades do dia-a dia). Neste último caso, empregam-se algoritmos de aprendizado de máquina que analisam o padrão dos sinais de aceleração (por exemplo, amplitude, variabilidade, potência, entropia etc) $)^{32,34,36}$.

- Tempo de uso: embora não haja um critério definitivo em relação ao tempo de uso para representar um dia válido, alguns pesquisadores têm adotado o mínimo de $10 \mathrm{~h} / \mathrm{dia}(600 \mathrm{~min} / \mathrm{dia})^{7,8}$, enquanto outros consideram períodos mais curtos de aproximadamente $8 \mathrm{~h} / \mathrm{dia}(480 \mathrm{~min} / \mathrm{dia})^{9,37}$. Em relação ao abandono parcial do acelerômetro, os registros de valor zero durante determinada sequência de tempo (por exemplo, 60 minutos de zeros consecutivos) podem ser adotados para detectar o tempo de não-uso ${ }^{7,38}$, embora não exista nenhuma diretriz que indique qual a melhor opção a ser utilizada. Por fim, o número de dias necessário para que um participante seja incluído na análise de dados varia nos estudos. Andersen et al. ${ }^{39}$ e Riddoch et al. ${ }^{40}$ utilizaram um mínimo de três dias, enquanto Cleland et al. ${ }^{37}$ utilizaram o mínimo de quatro dias. Em ambos os casos, incluiu-se ao menos um dia de fim de semana. No estudo de Davis e Fox ${ }^{41}$ adotou-se o mínimo de cinco dias para validação dos dados.

- Transformação de unidades de consumo de energia: estudos de revisão sobre a calibração dos acelerômetros ${ }^{24,42}$ evidenciaram a transformação dos dados de acelerômetro em diferentes métricas de AF. Um exemplo disso pode ser observado em estudos de calibração em crianças, em que diversos estudos desenvolveram algoritmos de calibração que predizem o consumo de oxigênio $\left(\mathrm{VO}_{2}\right)$, equivalente metabólico (METs), gasto energético total e gasto energético da $\mathrm{AF}(\mathrm{Kcals})^{28}$. Outro fator complicador desse processo é que a maioria dos algoritmos de calibração são específicos para cada marca de acelerômetro e localização do corpo nas quais foram originalmente validados ${ }^{16}$. Cada algoritmo de calibração é considerado válido apenas para populações semelhantes àquela 
da qual foi originalmente derivado. O método preferencial de estimar a AF por meio dos dados de acelerometria tem sido os pontos de corte para classificação do tempo gasto em diferentes intensidades de $\mathrm{AF}$ (comportamento sedentário, $\mathrm{AF}$ leve, AF moderada e AF vigorosa). Mais adiante são apresentados alguns dos pontos de corte comumente utilizados em crianças e adolescentes, adultos e idosos.

- Definição de bout para a AF: são períodos com durações pré-definidas (por exemplo, 5, 10 minutos sucessivos) e que potencialmente estão relacionados a implicações fisiológicas. O conceito de bout tem sido comumente aplicado a AF moderada a vigorosa (AFMV) ${ }^{43,44}$, mas as durações não tem sido consistentemente utilizadas na avaliação da AF. Quando o objetivo é verificar o atingimento das recomendações de AFMV, é recomendado que bouts contínuos de 10 minutos sejam utilizados, visto que as evidências de benefícios a saúde apontam neste sentido ${ }^{44}$.

Sugere-se, dessa forma, que as informações em relação a redução e manipulação dos dados sejam fornecidos nos estudos, especificando o número mínimo de dias em que o acelerômetro foi utilizado, o critério para considerar um dia válido, bem como os períodos prolongados de zeros nas contagens para determinar o abandono parcial ${ }^{45}$. Esses registros são de extrema importância para esclarecer os processos de tomada de decisões, justificar a análise final dos dados e os níveis de AF obtidos e relatados ${ }^{46}$, além de auxiliar nas comparações entre os estudos.

Atualmente, o uso dos acelerômetros para a mensuração dos níveis de AF tem sido muito debatido, tendo em vista a variedade de pontos de corte utilizados para classificar a intensidade da atividade. Essa diversidade, consequentemente, afeta a comparabilidade dos estudos ${ }^{14}$, incorpora erro nos dados, bem como classifica as pessoas em ativas ou inativas diferentemente ${ }^{47}$. Além de não haver conformidade a respeito dos limiares para counts de acelerômetros que identificam e classificam as atividades em sedentária, leve, moderada e vigorosa, os pontos de corte divergem para cada população ${ }^{24,42}$.

Portanto, a partir dos cuidados que devem ser tomados em cada fase, e das questões pontuadas acima, torna-se importante conhecer os pontos de corte mais comumente utilizados na literatura, a fim de reconhecer algumas de suas características. Neste sentido, apresentamos os dados que consideramos relevantes para crianças e adolescentes, adultos, idosos e populações especiais.

\section{Pontos de corte}

As Tabelas 1, 2 e 3 apresentam os principais pontos de corte para crianças e adolescentes, adultos e idosos, respectivamente. Cada tabela apresenta observações acerca das características gerais da amostra e do protocolo, método de desenvolvimento, escores de validade e confiabilidade, e os valores dos pontos de corte propriamente ditos. Ressalta-se que algumas informações referentes a Tabela 1 foram adaptadas do artigo de Romanzini et al. ${ }^{48}$, cujo objetivo principal foi revisar sistematicamente a validade dos pontos de corte para classificação da intensidade da AF em crianças e adolescentes.

Em crianças, existe evidência de que os pontos de corte desenvolvidos por Evenson et al. ${ }^{49}$ apresentam maior acurácia na classificação da intensidade da AF do que grande parte dos outros pontos de corte disponíveis ${ }^{50}$. Além disso, estudos também verificaram que a utilização de pontos de corte para classificação de epochs de $15 \mathrm{~s}$ apresentam performance superior aos pontos de corte para epochs 
TABELA 1 - Pontos de corte para classificação da intensidade da atividade física em crianças, pré-escolares e adolescentes a partir de acelerômetros posicionados no quadril.

\begin{tabular}{|c|c|c|c|c|}
\hline Autores & Amostra & Protocolo & Método de predição & Pontos de corte \\
\hline \multicolumn{5}{|c|}{ ActiGraph (Crianças e adolescentes) } \\
\hline Evenson et al. ${ }^{49}$ & $\begin{array}{l}\mathrm{n}=33 \text { ( } 5-8 \text { anos) } \\
\text { meninos e meninas. }\end{array}$ & $\begin{array}{l}\text { Atividades: sentado, assistir DVD, } \\
\text { colorir, caminhar }\left(3,2 \text { e } 4,8 \mathrm{~km} \cdot \mathrm{h}^{-1}\right) \text {, subir } \\
\text { degraus, dribles de basquete, pedalar, } \\
\text { polichinelos, correr }\left(6,4 \mathrm{~km} \cdot \mathrm{h}^{-1}\right) \text {. } \\
\text { Critério: calorimetria indireta. }\end{array}$ & $\begin{array}{l}\text { Curva ROC: } \\
\text { Áreas sob a curva } \\
\text { Sed }=0,98 \\
\text { Mod }=0,85 \\
\text { Vig }=0,83\end{array}$ & $\begin{array}{l}\text { Sed }=0-25 \text { counts } 15 \mathrm{~s}^{-1} \\
\text { Leve }=26-573 \text { counts } 15 \mathrm{~s}^{-1} \\
\text { Mod }=574-1002 \text { counts } 15 \mathrm{~s}^{-1} \\
\text { Vig } \geq 1003 \text { counts } 15 \mathrm{~s}^{-1}\end{array}$ \\
\hline Mattocks et al..$^{65}$ & $\begin{array}{l}\mathrm{n}=163 \text { ( } 12 \text { anos); } \\
\text { meninos e meninas. }\end{array}$ & $\begin{array}{l}\text { Atividades: repouso, jogar } \\
\text { videogame, caminhar (lento e } \\
\text { rápido), correr em ritmo próprio e } \\
\text { amarelinha. } \\
\text { Critério: calorimetria indireta. }\end{array}$ & $\begin{array}{l}\text { Regressão linear: } \\
\text { Dispêndio Energético }\left(\mathrm{k} \cdot \mathrm{kg}^{-1} \cdot \mathrm{min}^{-1}\right) \\
=-0,933+0,000098^{*} \text { counts } \mathrm{min}^{-1} \\
+0,091^{*} \text { idade(anos) }-0,0422^{*} \text { sexo } \\
\text { (masc }=0 \text {, fem }=1) \\
\text { Acurácia e precisão: } R^{2}=0,67 ; \text { Erro } \\
\text { padrão }=2,56 \times 10^{-6} \text { counts } \text { min }^{-1} \text {. }\end{array}$ & $\begin{array}{l}\text { Mod }=3581-6129 \text { counts } \text { min }^{-1} \\
\text { Vig } \geq 6130 \text { counts. } \text { min }^{-1}\end{array}$ \\
\hline Treuth et al. ${ }^{66}$ & $\begin{array}{l}\mathrm{n}=74(13-14 \text { anos); } \\
\text { apenas meninas. }\end{array}$ & $\begin{array}{l}\text { Atividades: repouso, ver TV, jogar } \\
\text { no computador, varrer, caminhar } \\
\left(4 \text { e } 5,6 \mathrm{~km} \cdot \mathrm{h}^{-1}\right) \text {, step, andar de } \\
\text { bicicleta }\left(19 \mathrm{~km} \cdot \mathrm{h}^{-1}\right) \text {, arremessos de } \\
\text { basquetebol, subir degraus, correr } \\
\left(8 \mathrm{~km} \cdot \mathrm{h}^{-1}\right) \text {. } \\
\text { Critério: calorimetria indireta. }\end{array}$ & $\begin{array}{l}\text { Regressão linear: } \\
\left.\text { MET }=2,01+0,00171 \text { (counts } 30 \mathrm{~s}^{-1}\right) \\
\left.\mathrm{MET}=2,01+0,00171 \text { (countsmin }{ }^{-1}\right) \\
\text { Acurácia e precisão: } \mathrm{R}^{2}=0,84 ; \text { Erro } \\
\text { padrão da estimativa }=1,36 \mathrm{METs} \text {. }\end{array}$ & $\begin{array}{l}\text { Sed }=0-50 \text { counts } 30 \mathrm{~s}^{-1} \\
\text { Leve }=51-1499 \text { counts } 30 \mathrm{~s}^{-1} \\
\text { Mod }=1500-2600 \text { counts } 30 \mathrm{~s}^{-1} \\
\text { Vig }>2600{\text { counts } 30 \mathrm{~s}^{-1}}^{-1}\end{array}$ \\
\hline Puyau et al. ${ }^{67}$ & $\begin{array}{l}n=26 \text { (6-16 anos); } \\
\text { meninos e meninas. }\end{array}$ & 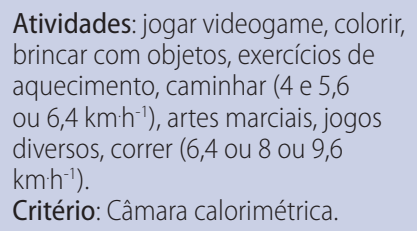 & $\begin{array}{l}\text { Dispêndio Energético }\left(\mathrm{kcal}^{\mathrm{kg}} \mathrm{kg}^{-1} \cdot \mathrm{min}^{-1}\right) \\
=0,0183+0,000010^{*} \text { counts } \mathrm{min}^{-1} \\
\text { Acurácia e precisão: } \mathrm{R}^{2}=0,75 ; \\
\text { Erro padrão da estimativa }=0,0172 \\
\mathrm{kcal}^{\mathrm{kg}}{ }^{-1} \cdot \mathrm{min}^{-1} \text {. }\end{array}$ & $\begin{array}{l}\text { Sed }=0-800 \text { counts } \text { min }^{-1} \\
\text { Leve }=800-3199 \text { counts } \mathrm{min}^{-1} \\
\text { Mod }=3200-8199 \text { counts } \mathrm{min}^{-1} \\
\text { Vig } \geq 8200 \text { countsmin }\end{array}$ \\
\hline \multicolumn{5}{|c|}{ ActiGraph (pré-escolares) } \\
\hline $\begin{array}{l}\text { Cauwenberghe } \\
\text { et al. }{ }^{51}\end{array}$ & $\begin{array}{l}n=18 \text { ( } 4-6 \text { anos) } \\
\text { meninos e meninas. }\end{array}$ & $\begin{array}{l}\text { Atividades: sentado, em pé, } \\
\text { desenhar, caminhar e correr na } \\
\text { esteira em } 7 \text { velocidades, caminhar } \\
\text { (fora do laboratório) e jogos livres. } \\
\text { Critério: observação direta. }\end{array}$ & $\begin{array}{l}\text { Curva ROC: } \\
\text { Áreas sob a curva } \\
\text { Sed }=0,95 \\
\text { Mod }=0,91 \\
\text { Vig }=0,94\end{array}$ & $\begin{array}{l}\text { Sed }=0-372 \text { counts } 15 \mathrm{~s}^{-1} \\
\text { Leve }=373-584 \text { counts } 15 \mathrm{~s}^{-1} \\
\text { Mod }=585-880 \text { counts } 15 \mathrm{~s}^{-1} \\
\text { Vig } \geq 881 \text { counts } 15 \mathrm{~s}^{-1}\end{array}$ \\
\hline Pate et al. ${ }^{68}$ & $\begin{array}{l}\mathrm{n}=30 \text { ( } 3-5 \text { anos) } \\
\text { meninos e meninas. }\end{array}$ & $\begin{array}{l}\text { Atividades: caminhar }(3,2 \text { e 4,8 } \\
\left.\mathrm{km}^{-1}\right) \text { e correr }\left(6,4 \mathrm{~km} \cdot \mathrm{h}^{-1}\right) \text {. } \\
\text { Critério: calorimetria indireta. }\end{array}$ & 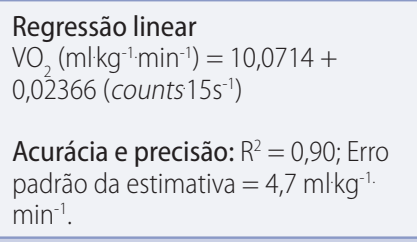 & $\begin{array}{l}\text { Mod }=420-841 \text { counts } 15 s^{-1} \\
\text { Vig } \geq 842 \text { counts } 15 s^{-1}\end{array}$ \\
\hline Sirard et al. ${ }^{69}$ & $\begin{array}{l}n=16(3-5 \text { anos }) \\
\text { meninos e meninas. }\end{array}$ & $\begin{array}{l}\text { Atividades: sentado, jogar sentado, } \\
\text { caminhada lenta e rápida, correr. } \\
\text { Critério: observação direta. }\end{array}$ & $\begin{array}{l}\text { Curva ROC: } \\
\text { Áreas sob a curva } \\
\text { Sed }=0,97-1,00 \\
\text { Mod }=0,92-0,97 \\
\text { Vig }=0,96-0,97\end{array}$ & $\begin{array}{l}\text { Sed }=0-398 \text { counts } 15 \mathrm{~s}^{-1} \\
\text { Leve }=399-890 \text { counts } 15 \mathrm{~s}^{-1} \\
\text { Mod }=891-1254 \text { counts } 15 \mathrm{~s}^{-1} \\
\text { Vig } \geq 1255 \text { countsmin }{ }^{-1}\end{array}$ \\
\hline Reilly et al. ${ }^{70}$ & $\begin{array}{l}\mathrm{n}=30 \text { ( } 3-4 \text { anos) } \\
\text { meninos e meninas. }\end{array}$ & $\begin{array}{l}\text { Atividades: não especificado. } \\
\text { Critério: observação direta. }\end{array}$ & $\begin{array}{l}\text { Curva ROC: } \\
\text { Sensibilidade: } 83 \pm 14 \% \text { (95\% IC: } \\
78 \text { - } 86 \% \text { ) } \\
\text { Especificidade: } 82 \pm 11 \% \text { (95\% IC: } \\
79-86 \% \text { ) }\end{array}$ & Sed $<1100$ countsmin ${ }^{-1}$ \\
\hline \multicolumn{5}{|c|}{ Actical (pré-escolares) } \\
\hline Evenson et al..$^{49}$ & $\begin{array}{l}\mathrm{n}=33 \text { ( } 5-8 \text { anos) } \\
\text { meninos e meninas. }\end{array}$ & $\begin{array}{l}\text { Atividades: sentado, ver DVD, } \\
\text { colorir, caminhar }\left(3,2 \text { e } 4,8 \mathrm{~km} \cdot \mathrm{h}^{-1}\right) \text {, } \\
\text { subir degraus }(88 \mathrm{bpm}) \text {, dribles de } \\
\text { basquetebol, polichinelos, pedalar, } \\
\text { correr }\left(6,4 \mathrm{~km} \cdot \mathrm{h}^{-1}\right) \text {. } \\
\text { Critério: calorimetria indireta. }\end{array}$ & $\begin{array}{l}\text { Curva ROC: } \\
\text { Áreas sob a curva } \\
\text { Sed }=0,98 \\
\text { Mod }=0,86 \\
\text { Vig }=0,86\end{array}$ & $\begin{array}{l}\text { Sed }=0-11 \text { counts } 15 \mathrm{~s}^{-1} \\
\text { Leve }=12-507 \text { counts } 15 \mathrm{~s}^{-1} \\
\text { Mod }=508-718 \text { counts } 15 \mathrm{~s}^{-1} \\
\text { Vig } \geq 719 \text { counts } 15 \mathrm{~s}^{-1}\end{array}$ \\
\hline
\end{tabular}




\begin{tabular}{|c|c|c|c|c|}
\hline Autores & Amostra & Protocolo & Método de predição & Pontos de corte \\
\hline Pfeiffer et al. ${ }^{71}$ & $\begin{array}{l}n=18 \text { ( } 3-5 \text { anos) } \\
\text { meninos e meninas. }\end{array}$ & 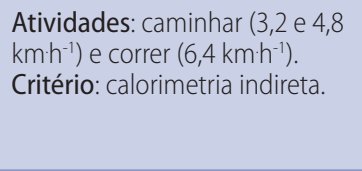 & $\begin{array}{l}\text { Regressão linear } \\
\mathrm{VO}_{2}=\text { counts } 15 \mathrm{~s}^{-1}(0,01437)+9,73 \\
\text { Acurácia e precisão: } \mathrm{R}^{2}=0,96 ; \text { Erro } \\
\text { padrão da estimativa }=3,02 \mathrm{ml} \cdot \mathrm{kg}^{-1} \text {. } \\
\mathrm{min}^{-1} \text {. }\end{array}$ & $\begin{array}{l}\text { Mod }=715-1410 \text { counts } 15 \mathrm{~s}^{-1} \\
\text { Vig } \geq 1411 \text { counts } 15 \mathrm{~s}^{-1}\end{array}$ \\
\hline Puyau et al. ${ }^{31}$ & $\begin{array}{l}n=32 \text { ( } 7-18 \text { anos); } \\
\text { meninos e meninas. }\end{array}$ & 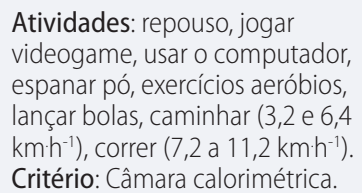 & $\begin{array}{l}\text { Regressão linear } \\
\left.\text { Dispêndio Energético (kcal } \mathrm{kg}^{-1} \cdot \mathrm{min}^{-1}\right) \\
=0,00423+0,00031^{*}{\text { counts } \mathrm{min}^{-1}} \\
(0,653) \\
\text { Acurácia: } \mathrm{R}^{2}=0,81 .\end{array}$ & $\begin{array}{l}\text { Sed }=0-100 \text { counts } \text { min }^{-1} \\
\text { Leve }=100-1499 \text { counts } \text { min }^{-1} \\
\text { Mod }=1500-6499 \text { counts } \text { min }^{-1} \\
\text { Vig } \geq 6500 \text { countsmin }\end{array}$ \\
\hline
\end{tabular}

Fonte: Adaptado de Romanzini et al. ${ }^{48}$.

TABELA 2 - Pontos de corte para classificação da intensidade da atividade física em adultos a partir de acelerômetros posicionados no quadril.

\begin{tabular}{|c|c|c|c|c|}
\hline Autores & Amostra & Protocolo & Método de predição & Pontos de corte \\
\hline \multicolumn{5}{|l|}{ ActiGraph } \\
\hline Freedson et al. ${ }^{30}$ & $\begin{array}{l}n=25 \text { homens }(24,8 \pm \\
4,2 \text { anos) e } 25 \text { mulheres } \\
(22,9 \pm 3,8 \text { anos). }\end{array}$ & $\begin{array}{l}\text { Atividades: caminhada e corrida } \\
\text { em esteira rolante em três } \\
\text { velocidades diferentes ( } 4,8 ; 6,4 \text { e } \\
\text { 9,7 } \mathrm{km}^{-1}{ }^{-1} \text {. } \\
\text { Critério: calorimetria indireta. }\end{array}$ & $\begin{array}{l}\text { Regressão linear } \\
\text { METs }=1,439008+ \\
\left(0,000795^{*} \text { countsmin }{ }^{-1}\right) \\
\text { Acurácia e precisão: } R^{2}=0,82 ; \\
\text { Erro padrão da estimativa }= \\
\pm 1,12 \text { METs }\end{array}$ & $\begin{array}{l}\text { Sed }^{*}=0-99 \text { countsmin } \\
\text { Leve 100-1951 countsmin } \\
\text { Mod }=1952-5724 \text { counts } \text { min }^{-1} \\
\text { Vig }=\geq 5725 \text { counts } \text { min }^{-1}\end{array}$ \\
\hline Sasaki et al. ${ }^{19}$ & $\begin{array}{l}\mathrm{n}=36 \text { ( } 27,8 \pm 8,6 \text { anos); } \\
\text { homens e mulheres. }\end{array}$ & $\begin{array}{l}\text { Atividades: caminhada e corrida } \\
\text { em esteira rolante em quatro } \\
\text { velocidades diferentes }(4,8 ; 6,4 ; \\
\left.9,7 \text { e } 12 \mathrm{~km} \cdot \mathrm{h}^{1}\right) \text {. } \\
\text { Critério: calorimetria indireta. }\end{array}$ & $\begin{array}{l}\text { Regressão linear } \\
\text { METs }=0,000863 \text { (VM3 counts } \\
\left.\text { min }^{-1}\right)+0,668876 \\
\text { Acurácia e precisão: } R^{2}=0,78 ; \\
\text { Erro padrão da estimativa }= \pm 1,3 \\
\text { METs } \\
\text { Obs. VM3 = vetor magnitude dos } \\
\text { dados triaxiais }\end{array}$ & $\begin{array}{l}\text { Sed }^{\#}=0-199 \text { countsmin } \\
\text { Leve }<2690 \text { countsmin } \\
\text { Mod }=2690-6166 \text { countsmin }^{-1} \\
\text { Vig }=\geq 6167 \\
\text { Obs. Os valores são aplicados } \\
\text { apenas a dados triaxiais (GT3X ou } \\
\text { superior). }\end{array}$ \\
\hline Hendelman et al. ${ }^{72}$ & $\begin{array}{l}\mathrm{n}=25(40,8 \pm 7,2 \\
\text { anos); } 10 \text { homens e } 15 \\
\text { mulheres. }\end{array}$ & 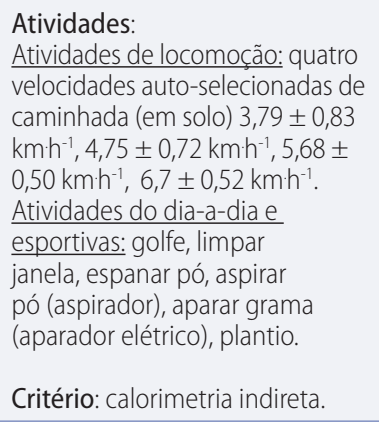 & $\begin{array}{l}\text { Regressão linear } \\
\text { METs }=2,922+ \\
\left(0,000409^{*} \text { countsmin }{ }^{-1}\right) \\
\text { Acurácia e precisão: } R^{2}=0,35 ; \\
\text { Erro padrão da estimativa }= \\
\pm 0,96 \mathrm{METs}\end{array}$ & $\begin{array}{l}\text { Sed }^{*}=0-99 \text { counts } \mathrm{min}^{-1} \\
\text { Leve }=100-190 \text { counts } \mathrm{min}^{-1} \\
\text { Mod }=191-7525 \text { counts } \mathrm{min}^{-1} \\
\text { Vig }>7525 \text { counts.min }\end{array}$ \\
\hline Swartz et al. ${ }^{73}$ & $\begin{array}{l}n=70 ; 31 \text { homens }(41 \pm \\
17 \text { anos) e } 39 \text { mulheres } \\
(42 \pm 14 \text { anos). }\end{array}$ & $\begin{array}{l}\text { Atividades: jardinagem, } \\
\text { atividade ocupacional } \\
\text { (caminhada e transporte } \\
\text { de peso), atividades do lar } \\
\text { (varrer, lavar louças, limpar o } \\
\text { chão, cozinhar, passar roupa } \\
\text { etc), cuidar de familiares, } \\
\text { atividades de condicionamento } \\
\text { (alongamento, calistenia, } \\
\text { caminhada lenta e rápida), } \\
\text { atividades de recreação (tênis } \\
\text { em duplas, golfe, softball) } \\
\text { Critério: calorimetria indireta. }\end{array}$ & $\begin{array}{l}\text { Regressão linear } \\
\text { METs }=2,606+ \\
\left(0,0006863^{*} \text { countsmin }{ }^{-1}\right) \\
\text { Acurácia e precisão: } R^{2}=0,317 \\
\text { Erro padrão da estimativa }= \\
\pm 1,16 \text { METs }\end{array}$ & 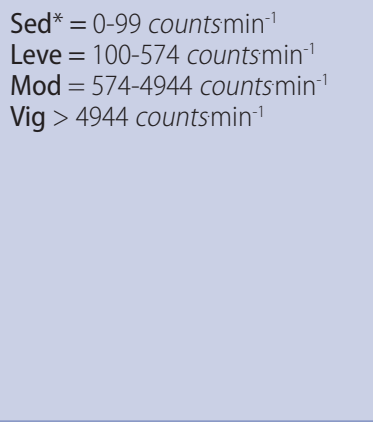 \\
\hline Brage et al. ${ }^{29}$ & $\begin{array}{l}n=12 \text { homens ( } 22,7- \\
30,0 \text { anos). }\end{array}$ & $\begin{array}{l}\left.\text { Atividades: Andar (3 e } 6 \mathrm{~km} \cdot \mathrm{h}^{-1}\right) \\
\text { e correr na esteira }(8,9,10,12 \\
\left.\text { 14, 16, } 18 \text { e } 20 \mathrm{~km} \cdot \mathrm{h}^{-1}\right)\end{array}$ & $\begin{array}{l}\text { Regressão linear } \\
\mathrm{VO}_{2} \mathrm{~kg}^{-1}=0,00260^{*} \text { counts } \mathrm{min}^{-1} \\
-0,07^{*} \text { fitness }+10,1\end{array}$ & $\begin{array}{l}\text { Sed }^{*}=0-99 \text { countsmin } \\
\text { Leve }=100-1809 \text { countsmin } \\
\text { Mod }=1810-5850 \text { counts } \text { min }^{-1} \\
\text { Vig }>5850 \text { countsmin }\end{array}$ \\
\hline
\end{tabular}




\begin{tabular}{|c|c|c|c|c|}
\hline Autores & Amostra & Protocolo & Método de predição & Pontos de corte \\
\hline Yngve et al. ${ }^{74}$ & $\begin{array}{l}\mathrm{n}=14 \text { homens }(23,7 \pm \\
2,6 \text { anos) e } 14 \text { mulheres } \\
(23,1 \pm 2,6 \text { anos })\end{array}$ & $\begin{array}{l}\text { Atividade: Caminhada e } \\
\text { corrida em velocidades auto- } \\
\text { selecionadas em pista indoor } \\
(4,3 \pm 0,4 ; 5,8 \pm 0,5 ; 9,6 \pm 0,7 \\
\mathrm{km}^{-1} \text { ) e esteira (baseadas nas } \\
\text { velocidades em pista). } \\
\text { Critério: calorimetria indireta. }\end{array}$ & $\begin{array}{l}\text { Regressão linear } \\
\text { Equação pista: } \\
\text { METs }=0 \text {, }\end{array}$ & $\begin{array}{l}\text { Sed }^{*}=0-99 \text { counts min }^{-1} \\
\text { Leve }=100-2631 \text { countsmin }^{-1} \\
\text { Mod }=2632-6404 \\
\text { countsmin } \\
\text { Vig }>6404 \text { countsmin }\end{array}$ \\
\hline \multicolumn{5}{|l|}{ Tritrac RT3 } \\
\hline Nichols et al. ${ }^{75}$ & $\begin{array}{l}\mathrm{n}=60 \text { ( } 23,4 \pm 2,9 \text { anos) } \\
\text { homens e mulheres. }\end{array}$ & $\begin{array}{l}\text { Atividades: caminhada e } \\
\text { corrida em esteira rolante em } \\
\text { três velocidades diferentes sem } \\
\text { inclinação }\left(3,2 ; 6,4 \text { e } 9,7 \mathrm{~km}^{-1}\right) \text { e } \\
\text { caminhada a } 6,4 \mathrm{~km} \cdot \mathrm{h}^{-1} \text { a } 5 \% \text { de } \\
\text { inclinação. } \\
\text { Critério: calorimetria indireta. }\end{array}$ & 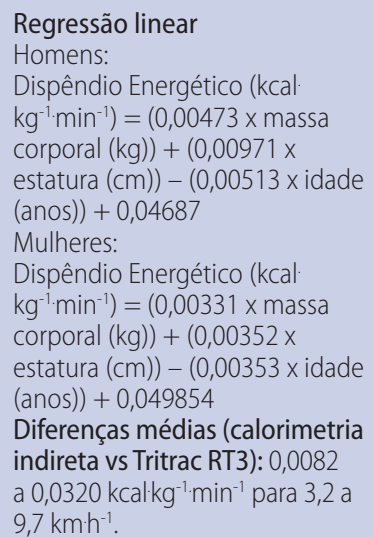 & $\begin{array}{l}2 \text { METs }=650 \text { unidades de } \\
\text { vetores } \\
4 \text { METs = } 1772 \text { unidades de } \\
\text { vetores } \\
7 \text { METs = } 3455 \text { unidades de } \\
\text { vetores }\end{array}$ \\
\hline
\end{tabular}

Legenda: Sed = comportamento sedentário; Leve = atividade física leve; Mod = atividade física moderada; Vig = atividade física vigorosa. * Ponto de corte para o comportamento sedentário originado de Matthews et al.24,76. \# O ponto de corte para derivação do comportamento sedentário a partir de dados triaxiais foi testado por Aguilar-Farías et al. ${ }^{77}$.

TABELA 3 - Pontos de corte para classificação da intensidade da atividade física em idosos acelerômetros posicionados no quadril.

\begin{tabular}{|c|c|c|c|c|}
\hline Autores & Amostra & Protocolo & Método de predição & Pontos de corte \\
\hline \multicolumn{5}{|l|}{ ActiGraph } \\
\hline Miller et al..$^{53}$ & $\begin{array}{l}n=90 \text {, ambos os sexos; } \\
3 \text { grupos de } 30 \\
20-29 \text { anos, } 40-49 \text { anos, } \\
\text { e } 60-69 \text { anos. }\end{array}$ & $\begin{array}{l}\text { Atividades: correr na esteira } \\
\text { ergométrica em diferentes } \\
\text { velocidades }\left(3,22 \mathrm{~km} \cdot \mathrm{h}^{-1} ; 4,83\right. \\
\mathrm{km} \cdot \mathrm{h}^{-1} ; 5,63 \mathrm{~km} \cdot \mathrm{h}^{-1} ; 6,44 \mathrm{~km} \cdot \mathrm{h}^{-1} \text {; } \\
9,66 \mathrm{~km} \cdot \mathrm{h}^{-1} ; 11,3 \mathrm{~km} \cdot \mathrm{h}^{-1} ; \mathrm{e} 12,9 \\
\left.\mathrm{~km} \cdot \mathrm{h}^{-1}\right) \text {. } \\
\text { Critério: calorimetria indireta. }\end{array}$ & $\begin{array}{l}\text { Regressão linear } \\
\text { METs }=1,532+\left(0,0007695^{*} \text { counts }\right. \\
\left.\text { min }^{-1}\right) \\
\text { Acurácia e precisão: } R^{2}=0,81 \text {; Erro } \\
\text { padrão da estimativa }= \pm 1,00 \text { METs }\end{array}$ & 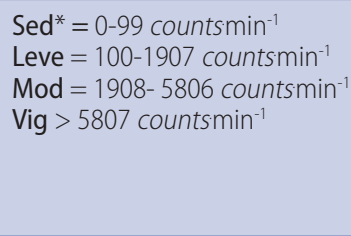 \\
\hline $\begin{array}{l}\text { Copeland e } \\
\text { Esliger }^{54}\end{array}$ & $\begin{array}{l}n=38, \text { idosos de ambos } \\
\text { os sexos }(69,7 \pm 3,5 \\
\text { anos). }\end{array}$ & $\begin{array}{l}\text { Atividades: caminhada em } \\
3 \text { diferentes velocidades em } \\
\text { uma esteira ergométrica }(2,4 \text {, } \\
\left.3,2 \text { e } 4,8 \mathrm{~km} \cdot \mathrm{h}^{-1}\right) \text {. } \\
\text { Critério: calorimetria indireta. }\end{array}$ & $\begin{array}{l}\text { Média de counts acima da } \\
\text { velocidade de } 3,2 \mathrm{~km} \cdot \mathrm{h}^{-1}\left(\mathrm{VO}_{2}=13,0\right. \\
\left. \pm 2,1 \mathrm{ml} \cdot \mathrm{kg}^{-1} \cdot \mathrm{min}^{-1}\right) \text {. }\end{array}$ & $\begin{array}{l}\text { Sed }^{*}=0-99 \text { countsmin } \\
\text { Leve }=100-1040 \text { counts } \text { min }^{-1} \\
\text { Mod }=1041 \text { countsmin }\end{array}$ \\
\hline
\end{tabular}

Legenda: Sed = comportamento sedentário; Leve = atividade física leve; Mod = atividade física moderada; Vig = atividade física vigorosa. * Ponto de corte para o comportamento sedentário originado de Matthews et al. ${ }^{24,76}$.

de maior duração ${ }^{51}$. Isso se deve a natureza intermitente das atividades realizadas por crianças e adolescentes. Sugere-se, portanto, que estudos com essa faixa etária priorizem os pontos de corte para epochs menores do que um minuto, pois esses métodos são mais adequados as atividades intermitentes e de curta duração.

Com relação a faixa etária adulta, os pontos de corte são altamente específicos em termos de performance. Aqueles pontos de corte desenvolvidos com atividades de locomoção são precisos na medida dessas mesmas atividades, mas tendem a subestimar a intensidade das atividades do dia-a-dia (por exemplo, varrer, passar pano, utilizar o aspirador de pó $)^{52}$. Por outro lado, os pontos de corte desenvolvidos com base em atividades do dia-a-dia classificam acuradamente tais atividades, mas superestimam a intensidade das atividades de locomoção ${ }^{52}$. A decisão de quais pontos de corte utilizar deve considerar tanto as atividades de interesse a serem medidas, como também o(s) estudo(s) com o qual(is) deseja-se realizar comparações. 
A faixa etária para qual as opções de pontos de corte são mais escassas é entre idosos. Esse segmento da população apresenta capacidade funcional reduzida em comparação a faixa etária adulta ${ }^{53}$. Isso justifica a utilização de valores menores para pontos de corte de classificação de AFMV. Neste sentido, Copeland et al. ${ }^{54}$ propuseram o valor de 1041 counts min $^{-1}$ como o limiar entre AF leve e AFMV. Apesar de estudos prévios terem utilizado pontos de corte para adultos em ido$\operatorname{sos}^{7,41}$, recomenda-se que pesquisadores considerem valores menores, como o proposto por Copeland et al. ${ }^{54}$, para classificar AFMV nessa faixa etária.

Com base nas tabelas e nas informações apresentadas acima, observa-se que existem diferenças e peculiaridades entre os pontos de corte. Devido à essas diferenças, sugere-se que os pesquisadores planejem a utilização de um determinado ponto de corte de acordo com as características de interesse da AF a serem estimadas, assim como da adequabilidade do método a amostra a ser estudada. A compararabilidade entre estimativas de $\mathrm{AF}$ de diferentes estudos depende dos pontos de corte aplicados. Existem evidências que a aplicação de diferentes pontos de corte em um mesmo banco de dados podem resultar em estimativas de AF altamente discrepantes ${ }^{47}$.

\section{Pontos de corte para populações especiais}

Os acelerômetros têm recebido crescente atenção no contexto de populações especiais. Estudos recentes empregaram acelerômetros na medida da AF em pacientes com doença de Parkinson ${ }^{55}$, doença pulmonar crônica obstrutiva - DPOC $^{56}$ e doença arterial periférica ${ }^{57}$. Esses estudos, no entanto, não aplicaram pontos de corte específicos às variações da $\mathrm{AF}$ ambulatorial e gasto energético resultantes das referidas condições. Os padrões locomotores alterados e a fisiopatologia das doenças impactam diretamente na $\mathrm{AF}$ ambulatória ${ }^{58}$. Essas alterações levam a dissociações entre os dados de acelerometria e o gasto energético das atividades ${ }^{59}$, o que demanda o desenvolvimento de pontos de cortes específicos para populações especiais.

As limitações de mobilidade também resultam em maior engajamento em atividades sedentárias e AF leves ${ }^{60}$. Algumas configurações dos acelerômetros podem interferir diretamente na sensibilidade para detecção dessas atividades de baixíssima intensidade. Em um estudo com pacientes idosos com doença de Parkinson, os autores buscaram comparar as estimativas de AF, obtidas por um acelerômetro ActiGraph ${ }^{\circledR} \mathrm{GT} 3 \mathrm{X}^{+}$, aplicando diferentes filtros na fase de aquisição de dados ${ }^{55}$. Os autores observaram que o filtro low frequency extension, quando comparado ao padrão, processou valores superiores para a média de counts e passos por dia, e para minutos em atividades de intensidade baixa e moderada, mas não para atividades vigorosas ${ }^{55}$. Porém, em uma amostra do National Health and Nutrition Examination Survey (NHANES), a AF medida por um acelerômetro ActiGraph ${ }^{\circledR} 7164$ foi superior em indivíduos saudáveis quando comparados a portadores de doença arterial periférica; nesse caso, a condição clínica representou uma redução de $23 \%$ da AF habitual ${ }^{60}$. Um estudo com pacientes portadores de DPOC revelou que acelerômetros de baixo custo, como o Fitbit ${ }^{\circledR}$ e o Personal Activity Monitor ${ }^{\circledR}$ AM300, apresentaram validade quando comparados ao SenseWear Armband ${ }^{\circledR}$ para o monitoramento da $\mathrm{AF}$ desses pacientes ${ }^{56}$.

Nesse contexto, padronizar os aspectos metodológicos da utilização de acelerômetros é importante para não enviesar as estimativas de AF, especialmente em indivíduos com limitações no padrão locomotor. Isto sugere a necessidade de pesquisas prévias para testar a validade dos acelerômetros e métodos de processamento de dados antes da implementação dos mesmos em estudos com popula- 
ções especiais.

\section{Comparabilidade entre acelerômetros de diferentes gerações}

O conhecimento sobre a comparabilidade dos dados provenientes de diferentes acelerômetros é imprescindível quando se deseja fazer comparações entre estudos e particularmente em análises de tendências dos níveis de AF. Muitos estudos são utilizados para tomada de decisões em políticas de saúde pública, sendo que estimativas incorretas a nível populacional podem influenciar diretamente essas decisões.

Resultados divergentes podem ser verificados ao analisar os dados de diferentes acelerômetros, mas essas disparidades podem ser observadas não apenas em acelerômetros de marcas distintas. Em aparelhos da mesma marca, mas de modelos diferentes, também são identificadas divergências nos dados obtidos ${ }^{19,61,62}$. As diferenças verificadas em testes mecânicos também são constatadas em ambiente $\operatorname{livre}^{62}$. Os acelerômetros mais antigos e mais novos podem diferir na resolução de conversão e taxa de amostragem, que são parâmetros que podem influenciar a saída dos dados ${ }^{21}$.

Em aparelhos da mesma marca porém de diferentes gerações, é possível verificar diferenças nos counts durante caminhada e corrida ${ }^{61,63}$, principalmente em velocidades muito lentas ${ }^{61}$ ou muito rápidas ${ }^{63}$. Parece não haver consenso na literatura se tais diferenças na contagem de counts seriam suficientes para acarretar divergências signiticativas nas classificações de intensidade da AF. Alguns estudos verificaram similaridade de counts em acelerômetros de mesma marca ${ }^{19}$, enquanto outros demonstraram diferenças em counts e nas estimativas de todas as intensidades de $\mathrm{AF}^{62}$.

A ativação da opção Low Frequency Extension (LFE) parece ser uma alternativa para reduzir as diferenças verificadas entre acelerômetros ActiGraph prévios e atuais, principalmente no que se refere ao comportamento sedentário e a atividades leves ${ }^{62}$. Em aparelhos de marcas diferentes, as evidências acerca da equivalência dos dados brutos de aceleração são escassas. Na comparação dos dados brutos dos sensores de movimento ActiGraph GT3X+ e GENEA, por exemplo, observou-se que a magnitude do vetor da aceleração foi significativamente diferente durante testes de oscilação mecânica em quatro frequências ${ }^{64}$. $\mathrm{O}$ acelerômetro GENEA produziu média de magnitude do vetor estatisticamente maior $(\mathrm{p}<0,05 ; 3,5$ para $6,2 \%)$ do que o GT3X+ em todas as frequências durante as oscilações ${ }^{64}$. Portanto, pode ser inadequado aplicar um modelo preditivo desenvolvido no GENEA aos dados coletados com o ActiGraph GT3X+.

\section{Considerações finais}

Tendo em vista todos os aspectos abordados neste estudo, recomendamos que os pesquisadores brasileiros considerem essas orientações em suas pesquisas. A utilização dos mais diversos métodos e formas de coletar, processar e analisar os dados de acelerometria têm dificultado as comparações entre estudos utilizando sensores de movimento. Nesse sentido, o maior relacionamento entre pesquisadores da área é necessário para garantir o avanço nas técnicas e nos métodos aplicados para mensurar a AF de forma objetiva. O conjunto de orientações aqui apresentado é uma iniciativa nessa direção e sua adoção pode facilitar a comparabilidade de resultados entre estudos com acelerômetros no Brasil.

\section{Contribuição dos autores}

J. E. Sasaki e T. R. B. Benedetti conceberam e planejaram o estudo. Todos os autores participaram na redação e edição do artigo, tendo aprovado a sua versão final. 


\section{Conflito de interesses}

Os autores declaram não ter nenhum conflito de interesse relacionado a publicação deste artigo.

\section{REFERÊNCIAS}

1. Warburton DER, Nicol CW, Bredin SSD. Health benefits of physical activity: the evidence. Can Med Assoc J. 2006;174(6):801-9.

2. Atienza AA, Moser RP, Perna F, Dodd K, Ballard-Barbash R, Troiano RP, et al. Selfreported and objectively measured activity related to biomarkers using NHANES. Med Sci Sports Exerc. 2011;43(5):815-21.

3. Hallal PC, Andersen LB, Bull FC, Guthold R, Haskell W, Ekelund U. Global physical activity levels: surveillance progress, pitfalls, and prospects. The Lancet. 2012;380(9838):247-57.

4. Chen KY, Bassett DR Jr. The technology of accelerometry-based activity monitors: current and future. Med Sci Sports Exerc. 2005;37(11 Suppl):S490-500.

5. Lee I-M, Shiroma EJ. Using accelerometers to measure physical activity in large-scale epidemiological studies: issues and challenges. Br J Sports Med. 2014;48(3):197-201.

6. Troiano RP, McClain JJ, Brychta RJ, Chen KY. Evolution of accelerometer methods for physical activity research. Br J Sports Med. 2014;48(13):1019-23.

7. Troiano RP, Berrigan D, Dodd KW, Mâsse LC, Tilert T, McDowell M. Physical activity in the United States measured by accelerometer. Med Sci Sports Exerc. 2008;40(1):181-8.

8. Davis MG, Fox KR, Hillsdon M, Sharp DJ, Coulson JC, Thompson JL. Objectively measured physical activity in a diverse sample of older urban UK adults. Med Sci Sports Exerc. 2011;43(4):647-54.

9. Ruiz JR, Ortega FB, Martínez-Gómez D, Labayen I, Moreno LA, De Bourdeaudhuij I, et al. Objectively measured physical activity and sedentary time in European adolescents: the HELENA study. Am J Epidemiol. 2011;174(2):173-84.

10. Hallal PC, Dumith S de C, Bastos JP, Reichert FF, Siqueira FV, Azevedo MR. Evolution of the epidemiological research on physical activity in Brazil: a systematic review. Rev Saúde Pública. 2007;41(3):453-60.

11. Tassitano RM, Bezerra J, Tenório MCM, Colares V, Barros MVG de, Hallal PC. Physical activity in brazilian adolescents: a systematic review. Braz J Kinanthropometry Hum Perform. 2007;9(1):55-60.

12. Bassett DR, Troiano RP, McClain JJ, Wolff DL. Accelerometer-based physical activity: total volume per day and standardized measures. Med Sci Sports Exerc. 2015;47(4):833-8.

13. Strath SJ, Kaminsky LA, Ainsworth BE, Ekelund U, Freedson PS, Gary RA, et al. Guide to the assessment of physical activity: Clinical and research applications: a scientific statement from the American Heart Association. Circulation. 2013;128(20):2259-79.

14. Silva I, Sasaki J, Gonçalves P. Mensuração da atividade física e tempo sedentário por meio de acelerômetros: cenário atual, perspectivas e demandas futuras. Rev Bras Atividade Física Saúde. 2016;21(4):293-6.

15. John D, Freedson P. ActiGraph and Actical physical activity monitors: a peek under the hood. Med Sci Sports Exerc. 2012;44(1 Suppl 1):S86-9.

16. Bassett DR Jr, Rowlands A, Trost SG. Calibration and validation of wearable monitors. Med Sci Sports Exerc. 2012;44(1 Suppl 1):S32-8.

17. Rothney MP, Apker GA, Song Y, Chen KY. Comparing the performance of three generations of ActiGraph accelerometers. J Appl Physiol. 2008;105(4):1091-7.

18. John D, Sasaki J, Hickey A, Mavilia M, Freedson PS. ActiGraphTM activity monitors: "the firmware effect." Med Sci Sports Exerc. 2014;46(4):834-9.

19. Sasaki JE, John D, Freedson PS. Validation and comparison of ActiGraph activity monitors. J Sci Med Sport. 2011;14(5):411-6.

20. Freedson P, Bowles HR, Troiano R, Haskell W. Assessment of physical activity using wearable monitors: recommendations for monitor calibration and use in the field. Med Sci Sports Exerc. 2012;44(1 Suppl 1):S1-4.

21. Sasaki JE, Da Silva KS, Da Costa BGG, John D. Measurement of Physical Activity Using Accelerometers. In: Computer-Assisted and Web-Based Innovations in Psychology, Special Education, and Health. 1st ed. Academic Press; 2016. p. 33-60. 
22. Trost SG, McIver KL, Pate RR. Conducting accelerometer-based activity assessments in field-based research. Med Sci Sports Exerc. 2005;37(11 Suppl):S531-43.

23. Ward DS, Evenson KR, Vaughn A, Rodgers AB, Troiano RP. Accelerometer use in physical activity: best practices and research recommendations. Med Sci Sports Exerc. 2005;37(11 Suppl):S582-8.

24. Matthews CE. Calibration of Accelerometer Output for Adults. Med Sci Sports Exerc. 2005;37(Supplement):S512-22.

25. Staudenmayer J, He S, Hickey A, Sasaki J, Freedson P. Methods to estimate aspects of physical activity and sedentary behavior from high-frequency wrist accelerometer measurements. J Appl Physiol Bethesda Md 1985. 2015;119(4):396-403.

26. Matthews CE, Hagströmer M, Pober DM, Bowles HR. Best practices for using physical activity monitors in population-based research. Med Sci Sports Exerc. 2012;44(1 Suppl 1):S68-76.

27. Shiroma EJ, Cook NR, Manson JE, Buring JE, Rimm EB, Lee I-M. Comparison of SelfReported and Accelerometer-Assessed Physical Activity in Older Women. PLOS ONE. 2015;10(12):e0145950.

28. Heil DP, Brage S, Rothney MP. Modeling physical activity outcomes from wearable monitors. Med Sci Sports Exerc. 2012;44(1 Suppl 1):S50-60.

29. Brage S, Wedderkopp N, Franks PW, Andersen LB, Froberg K. Reexamination of validity and reliability of the CSA monitor in walking and running. Med Sci Sports Exerc. 2003;35(8):1447-54.

30. Freedson PS, Melanson E, Sirard J. Calibration of the Computer Science and Applications, Inc. accelerometer. Med Sci Sports Exerc. 1998;30(5):777-81.

31. Puyau MR, Adolph AL, Vohra FA, Zakeri I, Butte NF. Prediction of activity energy expenditure using accelerometers in children. Med Sci Sports Exerc. 2004;36(9):1625-31.

32. Staudenmayer J, Pober D, Crouter S, Bassett D, Freedson P. An artificial neural network to estimate physical activity energy expenditure and identify physical activity type from an accelerometer. J Appl Physiol. 2009;107(4):1300-7.

33. Freedson PS, Lyden K, Kozey-Keadle S, Staudenmayer J. Evaluation of artificial neural network algorithms for predicting METs and activity type from accelerometer data: validation on an independent sample. J Appl Physiol. 2011;111(6):1804-12.

34. Zhang S, Rowlands AV, Murray P, Hurst TL. Physical activity classification using the GENEA wrist-worn accelerometer. Med Sci Sports Exerc. 2012;44(4):742-8.

35.Zhang S, Murray P, Zillmer R, Eston RG, Catt M, Rowlands AV. Activity classification using the GENEA: optimum sampling frequency and number of axes. Med Sci Sports Exerc. 2012;44(11):2228-34.

36. Sasaki JE, Hickey AM, Staudenmayer JW, John D, Kent JA, Freedson PS. Performance of Activity Classification Algorithms in Free-Living Older Adults. Med Sci Sports Exerc. 2016;48(5):941-50.

37. Cleland V, Crawford D, Baur LA, Hume C, Timperio A, Salmon J. A prospective examination of children's time spent outdoors, objectively measured physical activity and overweight. Int J Obes. 2008;32(11):1685-93.

38. Choi L, Ward SC, Schnelle JF, Buchowski MS. Assessment of wear/nonwear time classification algorithms for triaxial accelerometer. Med Sci Sports Exerc. 2012;44(10):2009-16.

39. Andersen LB, Harro M, Sardinha LB, Froberg K, Ekelund U, Brage S, et al. Physical activity and clustered cardiovascular risk in children: a cross-sectional study (The European Youth Heart Study). Lancet. 2006;368(9532):299-304.

40. Riddoch CJ, Mattocks C, Deere K, Saunders J, Kirkby J, Tilling K, et al. Objective measurement of levels and patterns of physical activity. Arch Dis Child. 2007;92(11):963-9.

41. Davis MG, Fox KR. Physical activity patterns assessed by accelerometry in older people. Eur J Appl Physiol. 2007;100(5):581-9.

42. Freedson P, Pober D, Janz KF. Calibration of accelerometer output for children. Med Sci Sports Exerc. 2005;37(11 Suppl):S523-30.

43. Haskell WL, Lee I-M, Pate RR, Powell KE, Blair SN, Franklin BA, et al. Physical activity and public health: updated recommendation for adults from the American College of Sports Medicine and the American Heart Association. Med Sci Sports Exerc. 2007;39(8):1423-34. 
44. US Department of Health and Human Services. 2008 Physical Activity Guidelines for Americans [Internet]. 2008 [Acessado 2015 Jul 14]. Disponível em: www.health.gov/ paguidelines

45. Ridgers ND, Fairclough S. Assessing free-living physical activity using accelerometry: Practical issues for researchers and practitioners. Eur J Sport Sci. 2011;11(3):205-13.

46. Esliger DW, Copeland JL, Barnes JD, Tremblay MS. Standardizing and Optimizing the Use of Accelerometer Data for Free-Living Physical Activity Monitoring. J Phys Act Health. 2005;2(3):366-83.

47. Watson KB, Carlson SA, Carroll DD, Fulton JE. Comparison of accelerometer cut points to estimate physical activity in US adults. J Sports Sci. 2014;32(7):660-9.

48. Romanzini M, Petroski EL, Reichert FF. Accelerometers thresholds to estimate physical activity intensity in children and adolescents: a systematic review. Rev Bras Cineantropom Desempenho Hum. 2012;14(1):101-13.

49. Evenson KR, Catellier DJ, Gill K, Ondrak KS, McMurray RG. Calibration of two objective measures of physical activity for children. J Sports Sci. 2008;26(14):1557-65.

50. Trost SG, Loprinzi PD, Moore R, Pfeiffer KA. Comparison of accelerometer cut points for predicting activity intensity in youth. Med Sci Sports Exerc. 2011;43(7):1360-8.

51. van Cauwenberghe E, Labarque V, Trost SG, de Bourdeaudhuij I, Cardon G. Calibration and comparison of accelerometer cut points in preschool children. Int J Pediatr Obes. 2011;6(2-2):e582-9.

52. Crouter SE, Churilla JR, Bassett DR Jr. Estimating energy expenditure using accelerometers. Eur J Appl Physiol. 2006;98(6):601-12.

53. Miller NE, Strath SJ, Swartz AM, Cashin SE. Estimating absolute and relative physical activity intensity across age via accelerometry in adults. J Aging Phys Act. 2010;18(2):158-70.

54. Copeland JL, Esliger DW. Accelerometer assessment of physical activity in active, healthy older adults. J Aging Phys Act. 2009;17(1):17-30.

55. Wallén MB, Nero H, Franzén E, Hagströmer M. Comparison of two accelerometer filter settings in individuals with Parkinson's disease. Physiol Meas. 2014;35(11):2287-96.

56. Vooijs M, Alpay LL, Snoeck-Stroband JB, Beerthuizen T, Siemonsma PC, Abbink JJ, et al. Validity and usability of low-cost accelerometers for internet-based self-monitoring of physical activity in patients with chronic obstructive pulmonary disease. Interact J Med Res. 2014;3(4):e14.

57. Loprinzi PD, Abbott K. Association of diabetic peripheral arterial disease and objectivelymeasured physical activity: NHANES 2003-2004. J Diabetes Metab Disord. 2014;13:63.

58. van Brussel M, van der Net J, Hulzebos E, Helders PJM, Takken T. The Utrecht approach to exercise in chronic childhood conditions: the decade in review. Pediatr Phys Ther. 2011;23(1):2-14.

59. Motl RW, Snook EM, Agiovlasitis S, Suh Y. Calibration of accelerometer output for ambulatory adults with multiple sclerosis. Arch Phys Med Rehabil. 2009;90(10):1778-84.

60. Loprinzi PD, Sheffield J, Tyo BM, Fittipaldi-Wert J. Accelerometer-determined physical activity, mobility disability, and health. Disabil Health J. 2014;7(4):419-25.

61. Kozey SL, Staudenmayer JW, Troiano RP, Freedson PS. Comparison of the ActiGraph 7164 and the ActiGraph GT1M during self-paced locomotion. Med Sci Sports Exerc. 2010;42(5):971-6.

62. Ried-Larsen M, Brønd JC, Brage S, Hansen BH, Grydeland M, Andersen LB, et al. Mechanical and free living comparisons of four generations of the Actigraph activity monitor. Int J Behav Nutr Phys Act. 2012;9:113.

63. John D, Tyo B, Bassett DR. Comparison of four ActiGraph accelerometers during walking and running. Med Sci Sports Exerc. 2010;42(2):368-74.

64. John D, Sasaki J, Staudenmayer J, Mavilia M, Freedson PS. Comparison of raw acceleration from the GENEA and ActiGraphTM GT3X+ activity monitors. Sensors. 2013;13(11):14754-63.

65. Mattocks C, Leary S, Ness A, Deere K, Saunders J, Tilling K, et al. Calibration of an accelerometer during free-living activities in children. Int J Pediatr Obes. 2007;2(4):218-26.

66. Treuth MS, Schmitz K, Catellier DJ, McMurray RG, Murray DM, Almeida MJ, et al. Defining accelerometer thresholds for activity intensities in adolescent girls. Med Sci Sports Exerc. 2004;36(7):1259-66. 
67. Puyau MR, Adolph AL, Vohra FA, Butte NF. Validation and calibration of physical activity monitors in children. Obes Res. 2002;10(3):150-7.

68. Pate RR, Almeida MJ, McIver KL, Pfeiffer KA, Dowda M. Validation and calibration of an accelerometer in preschool children. Obes Silver Spring Md. 2006;14(11):2000-6.

69. Sirard JR, Trost SG, Pfeiffer KA, Dowda M, Pate RR. Calibration and Evaluation of an Objective Measure of Physical Activity in Preschool Children. J Phys Act Health. 2005;2(3):345-57.

70. Reilly JJ, Coyle J, Kelly L, Burke G, Grant S, Paton JY. An objective method for measurement of sedentary behavior in 3- to 4-year olds. Obes Res. 2003;11(10):1155-8.

71. Pfeiffer KA, McIver KL, Dowda M, Almeida MJCA, Pate RR. Validation and calibration of the Actical accelerometer in preschool children. Med Sci Sports Exerc. 2006;38(1):152-7.

72. Hendelman D, Miller K, Baggett C, Debold E, Freedson P. Validity of accelerometry for the assessment of moderate intensity physical activity in the field. Med Sci Sports Exerc. 2000;32(9 Suppl):S442-9.

73. Swartz AM, Strath SJ, Bassett DR Jr, O’Brien WL, King GA, Ainsworth BE. Estimation of energy expenditure using CSA accelerometers at hip and wrist sites. Med Sci Sports Exerc. 2000;32(9 Suppl):S450-6.

74. Yngve A, Nilsson A, Sjostrom M, Ekelund U. Effect of monitor placement and of activity setting on the MTI accelerometer output. Med Sci Sports Exerc. 2003;35(2):320-6.

75. Nichols JF, Morgan CG, Sarkin JA, Sallis JF, Calfas KJ. Validity, reliability, and calibration of the Tritrac accelerometer as a measure of physical activity. Med Sci Sports Exerc. 1999;31(6):908-12.

76. Matthews CE, Chen KY, Freedson PS, Buchowski MS, Beech BM, Pate RR, et al. Amount of time spent in sedentary behaviors in the United States, 2003-2004. Am J Epidemiol. 2008;167(7):875-81.

77. Aguilar-Farías N, Brown WJ, Peeters GMEEG. ActiGraph GT3X+ cut-points for identifying sedentary behaviour in older adults in free-living environments. J Sci Med Sport. 2014;17(3):293-9.

Endereço para

Correspondência

Tânia R. Bertoldo Benedetti

tania.benedetti@ufsc.br
Rua Mediterrâneo, 204, apto 501,

Córrego Grande, Florianópolis, Santa

Catarina. CEP:88037-610

Fone (48) $9924-2990$
RECEBIDO

REVISADO

$22 / 01 / 2016$

$19 / 08 / 2016$

$03 / 01 / 2017$

APROVADO $\quad 04 / 01 / 2017$ 